\title{
Increasing communication between a man and a dog
}

\author{
Germain Lemasson $^{1}$, Sylvie Pesty ${ }^{2}$, Dominique Duhaut ${ }^{1}$ \\ ${ }^{1}$ University of South-Brittany \\ UMR 6285 - Lab-STICC \\ E-mail: \{germain.lemasson, dominique.duhaut\}@univ-ubs.fr \\ ${ }^{2}$ Universty of Grenoble \\ UMR 5217- LIG \\ sylvie.pesty@imag.fr
}

\begin{abstract}
In this paper, we present the first results we have concerning our ongoing work on a robotic system embedded on a dog to enrich communication. Two problems are addressed here: How to keep control of a dog when the human does not see it? For dog trained to do some specific activities in particular situation, how to detect this activity? We present here results on controlling the $\operatorname{dog}$ by an embedded voice and a real-time recognition of some activities of the dog : walk, seat, run, lying.
\end{abstract}

\section{INTRODUCTION}

The interaction between man and machine is widely studied and how technology can strengthen the bond between people [1][2]. Our team is more interested in the communication between the machine and the animal especially with a dog. And how it can help or improve communication between the dog and his owner. Indeed dogs are loyal companion of humans since 14000 years. Humans and dogs have evolved together [3][4]. Man have first trained dogs in order to help him in is task, hunting dog, shepherd dog. Relatively recently appeared a new type of dog, the service dog. The first ones was trained after WWI for blind soldiers; it is the guide dog that we know today [5].And more recently dogs are trained for assisting person with reduced mobility. These dogs can help people in their daily life; they are also an extraordinary psychological support. The dog is also with the cat the most popular companion animals. He is considered as a member of the family or as a close friend.

In this paper we present our works one how we can communicate with dog through technological equipment.

\section{RELATED WORKS}

The development of technology for animals is just starting. Several products localization begins to appear on the market. [6] Golbeck et al conducted a study using a computer to communicate with a dog. Ribeiro et al. [7] proposed a method to detect the pose of USAR dogs. It uses two 1- axis accelerometer to find the poses of USAR dogs. There method detects the transition between the poses. Results on static activity are really encouraging. [8] Paldanius et al. conducted a study to understand the experiences and expectations of dog owners for communication technology. How they use existing equipment as GPS location. Vernay et al. [9] have imagined scenario for assistance dog. Our team works with this type of $\operatorname{dog}$ and is based these scenario.

\section{COMMUNICATION WITH A DOG}

\section{A. Context}

Our team works in conjunction with the French association Handi'Chien. The Handi'Chien association has trained more than 1,000 dogs in 20 years and getting more and more requests each year. These dogs are trained from birth, and the training lasts two years. The first 6 months of their life, they spend it in a foster family where they socialize and learn basic commands. Then, for 18 months, they are trained in specialized centers where they learn fifty orders. At the end of their training, they are given for free to their new owner. The persons requesting a dog are evaluated to see if they can take care of the dog, they also received a short training on how to act with their dogs. Despite of this preliminary work, some problem may occur. Vernay et al. [9] exposes the problem and propose solution using technology. We based our work from these scenarios. The first scenario proposes a solution to the problem of the recall that arises when the dog is not in range of sight or of voice. This can happen if the dog has escaped the attention of the owner (pursuit of a cat) or simply if the $\operatorname{dog}$ is in pause; it is allowed to move away from its owner. The dog must return to its owner. In the case of a person with a disability, it does not necessarily have the voice power and mobility necessary to regain the dog's attention. In this situation a robotic system can serve as an intermediary between the owner and his dog. This type of situation occurs outdoor in open fields. This means the robotic system must be carried by the dog to communicate with him wherever he goes.

\section{B. Communicating with the dog}

Communication is something that is done in both directions. On one hand we have information transmission from the owner to the dog and on the other hand we have information transmission from the dog to the owner.

In order to send information to the dog, we consider 5 channels which are the five senses: view, hearing, smell, touch, taste. We thought of means on how to stimulate each of his sense. Tab. 1 shows some of the means we plan to use. 
The order of importance of the senses in dogs is different from the order in humans. The most important sense of the dog is the smell then the hearing and only in third position the view which are our first sense. Dogs communicate between them using smell pheromone. But it's not how we communicate and using this channel is not easy. We can still use it like with some anti-bark collar equipped with spray of lemongrass which is an odor dogs hate. Our best sense, the view in our context is also not the best way to communicate. We can't put a screen in front of the dog and dogs are shortsighted. However dogs are really receptive to pointing, like when you point an object with your finger. It also works with laser pointer so it's a good option. Hearing is our common second best sense we both use it to communicate and dogs understand humans speaking they can memorized 50 orders, plus some name of object. Hearing is definitely the best way to communicate with a dog. So this is the first sense we try to use to communicate with the dog.

\begin{tabular}{|l|l|}
\hline Sense & Technical means \\
\hline Smell & Spray \\
\hline Hearing & $\begin{array}{l}\text { Vocal order, } \\
\text { ultrasound }\end{array}$ \\
\hline View & laser pointer \\
\hline Touch & $\begin{array}{l}\text { Cuddle, } \\
\text { Vibration }\end{array}$ \\
\hline Taste & Food \\
\hline \multicolumn{2}{|c|}{ Tab. 1. Sense and possible action } \\
\hline
\end{tabular}

The other part of the communication is getting information from the dog to the owner. The first information that can be useful for the owner is where is his dog if is not next to him. The dog might be hidden by a corner of a street or in a park he may be one or two hundred meters ahead from him. A second information is how is the environment of the dog. Is there anything that keeps is attention? Other animals or people? A third information is the activity of the dog. What is he doing, is he running or just laying down ? We think these three information allow the owner to know the situation of his dog so he can send new orders or just see if his dog understand the previous one.

\section{Realisation}

We develop two prototypes. The first prototype is a harness equiped with speakers connected to a smartphone fasten too the harness Fig. 4. Fig. 5.. The owner of the dog recorded basic order: "seat", "lay down", "to your place" and "good" for the gratification when the dog respect an order. We use recorderd orders and not generatred voice in order to keep good intonations. They are put on the smartphone one the back of the dog. Then the smartphone is connected to two amplified speakers fixed to the harness below the ears of the dog. The vocal orders can be triggered remotly with a second smartphone. The two smartphones run on androids and communicates using the standard Wi-fi Direct which allow us a distance of 50 meters between the two smartphone without too much delay. This prototype use only vocal order however the next one will combine more chanel. We think it's primordial for the system to make physical gratiphication, like vibration or simulated cuddle.

We made a second prototype to detect the activity of the dog. We also use two smartphone for this prototype but this time we use the 3-axis accelerometer and 3-axis gyroscope sensors. The smartphone is placed on the back of the dog. The orientation of the sensors is shown in Fig. 1. Y to the dog's head, $\mathrm{X}$ to his right side and $\mathrm{Z}$ perpendicular to his back.

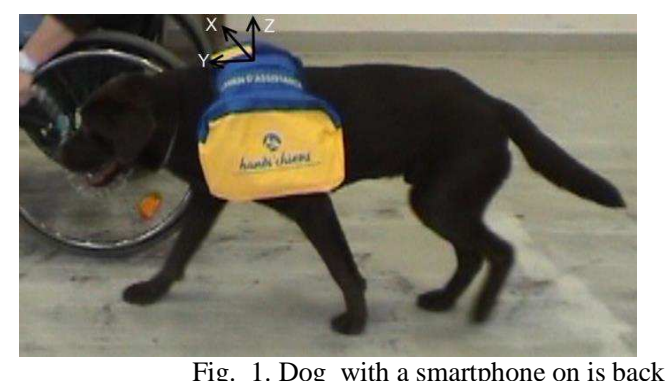

Fig. 1. Dog with a smartphone on is back.

We choose to start by detecting four basic activities, sitting, laying down, running, walking. Fig. 2. describes the data flowchart of the presented method. Data are collected by the smartphone 1 on the back of the dog then sent to the smartphone 2 in the hand of the owner of the dog which performs the detection.

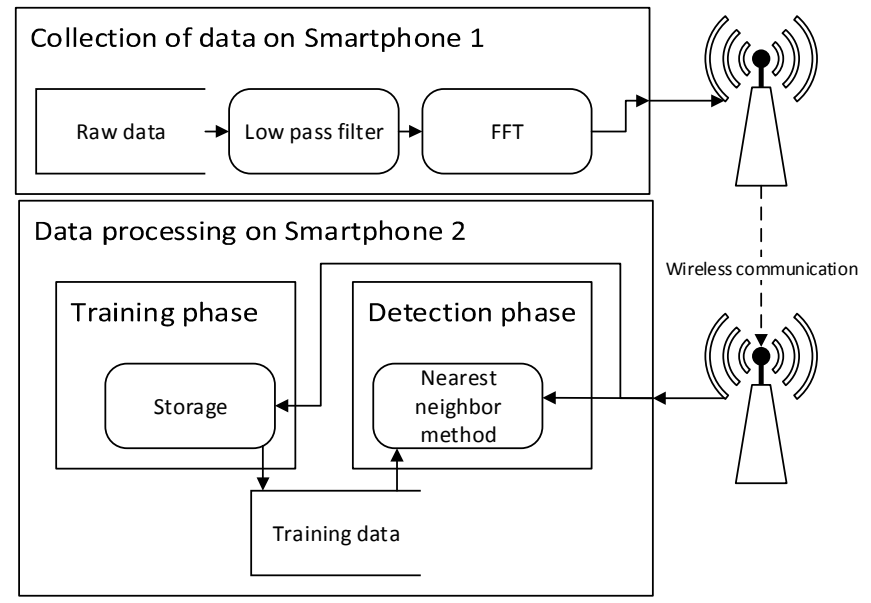

Fig. 2. Data flowchart

After a first data collection we conclude that the accelerometer $\mathrm{z}$-axis and the gyro $\mathrm{z}$-axis are sufficient to determine our four basic activities. The single $\mathrm{z}$-axis accelerometer can even be sufficient but we chose to add the $\mathrm{z}$-axis gyroscope for more reliability.

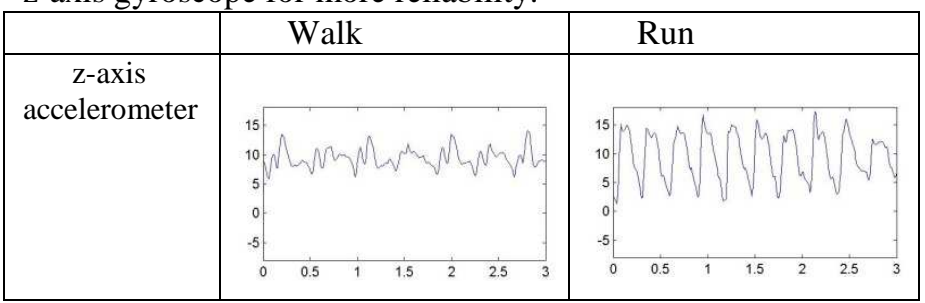




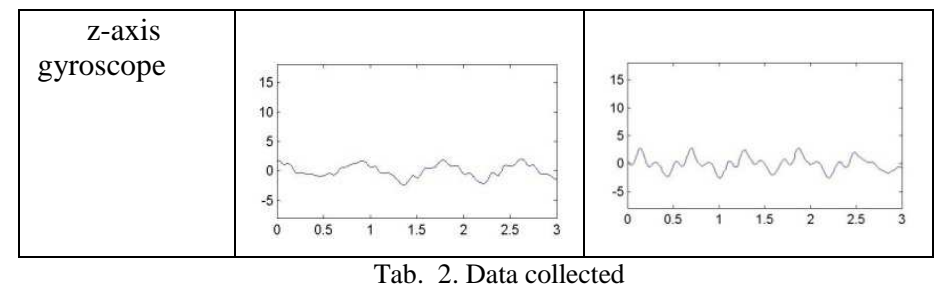

Tab. 2. shows the collected data. The $\mathrm{x}$-axis of the curves is the times in second ( 3 seconds) and the y-axis is the values returned by the z-axis of the sensors. We clearly see the periodicity of the signal. We use a low pass filter and a FFT (Fast Fourier Transform) [10] with a two second window to get the maximum amplitude and the periodicity of the signal. For the sitting and the running the signal are flat. However for the sitting activity, the mean of the signal is less than $G$ the gravitational constant, unlike the 3 others activities where the means are close to $\mathrm{G}$. These data are sent to the smartphone of the owner. We use the method of the nearest neighbor, a supervised learning method. In a fist phase we know the activities corresponding of the data and we label it. At the beginning of the detection phase, the training data are scaled and placed in a 5-dimensional space. Then each received data is scaled and we use the Minkowski distance (generalization of the Euclidean distance) to find its nearest neighbor. The activity is determined by the activity of its nearest neighbor.

For the positioning problem we use the A-GPS service available on the smartphone on the back of the dog and send the position to the smartphone of the owner which displays it.

Fig. 3. shows the interface of the owner.

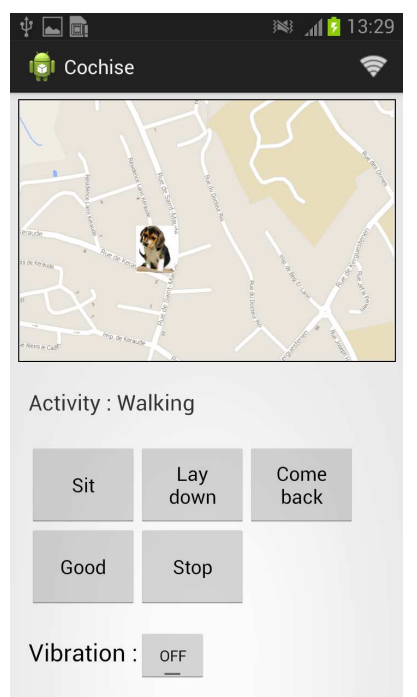

Fig. 3. Interface of the owner

\section{EXPÉRIMENTATIONS AND RESULTS}

The aim of the experimentation with the prototype using speakers is to see if the dog will obey to the recorded order if is not in eye contact with is owner. The orders are recorded using the voice of the owner. We put the dog alone in a room where we place webcams to see the reaction of the dog and a pillow that he recognizes as is place. Then we triggered the order. The first order was sit down, at first the reaction of the dog was astonishment, he didn't understand where the voice coming from and searched his owner. We triggered the order two more times and he finally sit. We repeat the experience with the other orders. Each time we must triggered the orders two or three time to be obeyed but he was less and less surprised. These results are really encouraging for our project.
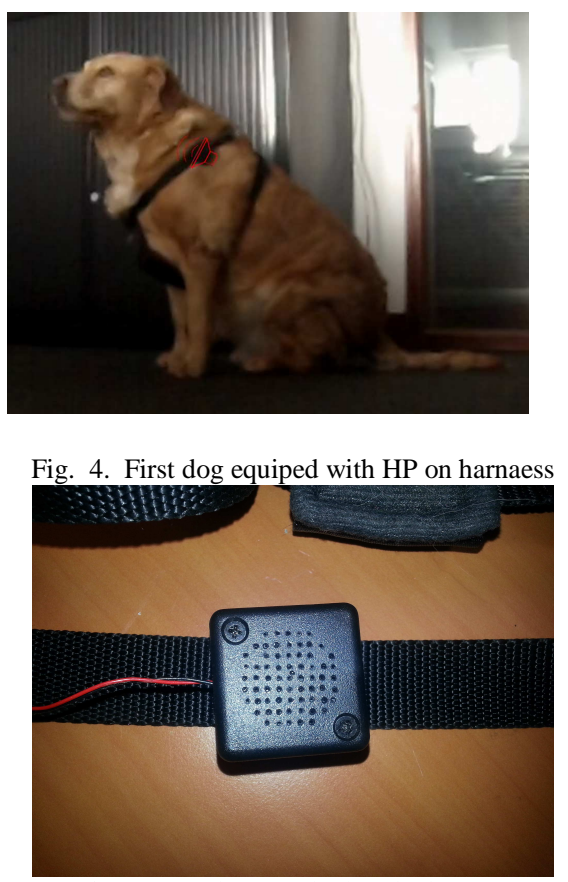

Fig. 5. Speaker attached to the harness

In the experiment with the prototype for the activity detection, we tested our method for 8 minutes during which we ask the dog to walk, run, sit down and lie down. The activity changed every 30 seconds.

\begin{tabular}{|c|l|l|l|}
\hline Activity & Correct & Incorrect & \\
\hline Walk & 55 & 5 & $91 \%$ \\
\hline Race & 50 & 10 & $83 \%$ \\
\hline $\begin{array}{c}\text { Sitting } \\
\text { position }\end{array}$ & 52 & 8 & $86 \%$ \\
\hline $\begin{array}{c}\text { Lying } \\
\text { position }\end{array}$ & 45 & 15 & $75 \%$ \\
\hline
\end{tabular}

Tab. 3. shows the result our experimentation. Errors in the lying position are mainly due to the position of the smartphone on the harness. Indeed, when the dog was lying but lifts high enough his head the harness and the smartphone fell slightly like if the dog was sitting down. The overall percentage of detection on our experiment is $83 \%$.

\section{CONCLUSION}

In this paper, we introduce our first ideas on how a system can communicate with a dog. With the experiments done with our prototypes we see that a dog can obey to registered vocal order out without the presence of his master and we can detect remotely the activity of the dog. We are actually working on a new harness which combines multiple stimuli: vibration, voice and physical pressure. This new is in test with he educators and we expect results soon. Another important part of our 
works will be the design of the software on the side of the user. As indicated by [8], the importance of the user interface is really important. It must be easy to use and meets the expectation of the user. In our case we have two type of user: the dog educators and the disabled people. The interface must be adapted to each type of disease.

\section{ACKNOWLEDGMENT}

This work is supported by French National Research Agency (ANR-012-BLAN).

\section{REFERENCES}

[1] T. K. Judge, C. Neustaedter, S. Harrison, and A. Blose, "Family portals: connecting families through a multifamily media space," in Proceedings of the SIGCHI Conference on Human Factors in Computing Systems, New York, NY, USA, 2011, pp. 1205-1214.

[2] T. K. Judge and C. Neustaedter, "Sharing conversation and sharing life: video conferencing in the home," in Proceedings of the SIGCHI Conference on Human Factors in Computing Systems, New York, NY, USA, 2010, pp. 655-658.

[3] A. Prestrude, "Dogs in service to humans," Comp. Psychol. Handb., pp. 386-392, 1998.

[4] C. A. Driscoll, D. W. Macdonald, and S. J. O'Brien, "From wild animals to domestic pets, an evolutionary view of domestication," Proc. Natl. Acad. Sci. U. S. A., vol. 106, no. Suppl 1, pp. 9971-9978, Jun. 2009.
[5] E. Weiss and G. Greenberg, "Service dog selection tests: Effectiveness for dogs from animal shelters," Appl. Anim. Behav. Sci., vol. 53, no. 4, pp. 297-308, Jul. 1997.

[6] J. Golbeck and C. Neustaedter, "Pet video chat: monitoring and interacting with dogs over distance," in CHI '12 Extended Abstracts on Human Factors in Computing Systems, New York, NY, USA, 2012, pp. 211-220.

[7] C. Ribeiro, A. Ferworn, M. Denko, and J. Tran, "Canine Pose Estimation: A Computing for Public Safety Solution," in Canadian Conference on Computer and Robot Vision, 2009. CRV '09, 2009, pp. 37 -44.

[8] M. Paldanius, T. Kärkkäinen, K. Väänänen-VainioMattila, O. Juhlin, and J. Häkkilä, "Communication technology for human-dog interaction: exploration of dog owners' experiences and expectations," in Proceedings of the SIGCHI Conference on Human Factors in Computing Systems, New York, NY, USA, 2011, pp. 2641-2650.

[9] D. Vernay, P. Rybarczyk, M.-C. Lebret, and Y. Rybarczyk, "Collaboration hommes, chiens et robots: quels scenarios?," 2011.

[10] R. N. Bracewell, The Fourier transform and its applications, vol. 31999. . 